\title{
Results and future perspectives of the neutron spin structure studies at Jefferson Lab
}

\author{
A. DEUR*) \\ University of Virginia
}

The Jefferson Lab Hall A polarized ${ }^{3} \mathrm{He}$ (neutron spin structure) program is presented with a special focus on the measurement of the $Q^{2}$ evolution of the generalized GDH integral.

\section{Introduction}

Starting in the late 70's the availability of polarized beams and targets have allowed further checks of QCD in its perturbative regime and the measurement of the nucleon spin structure functions in this domain. These experiments, which have taken place at high energy laboratories such as CERN, DESY and SLAC, provided a fair understanding of the strong interaction in the perturbative domain ( $\mathrm{pQCD}$ ). Of lower energy ( 0.8 to $6 \mathrm{GeV}$ ), the new accelerator at Jefferson Lab is characterized by its continuous, highly polarized, electron beam. It is complementing the study of the nucleon structure and of QCD by allowing precise studies of the transition region from the parton to the nuclear degrees of freedom of the strong interaction. The transition between these two regimes is far from understood and is a key issue in our understanding of the strong interaction. I will present in this talk the Jefferson Lab Hall A $\overrightarrow{3} \mathrm{He}$ program which aims to provide a set of high quality data on neutron spin structure in the transition domain as well as in the large $\mathrm{x}$ domain, difficult to achieve for higher energy machines. The first completed experiment (measurement of the $Q^{2}$ evolution of the generalized GDH integral) will be the central part of this talk. I will then briefly present the other currently running or future experiments (higher twist measurements and large $x$ physics).

\section{The experimental setup}

\subsection{The Hall A high resolution spectrometers}

The experiments mentioned above are measuring the inclusive ${ }^{3} \overrightarrow{\mathrm{He}}\left(e, e^{\prime}\right) X$ reaction. They took or will take place in Jefferson Lab Hall A [1]. The hall contains two nearly identical High Resolution Spectrometers (HRS). In our case, they are used independently (inclusive reactions) and in symmetric settings. The comparison of the data from both arms reduces and measures our systematic errors. The HRS detector package consists of a set of wire chambers for tracking and high resolution momentum measurement $\left(\Delta \mathrm{p} / \mathrm{p} \simeq 10^{-4}\right)$, two planes of scintillators for data acquisition triggering and a set of preshower, shower and Cerenkov detectors for particle identification ( $\pi$ rejection inefficiency at the few $10^{-4}$ level).

*) For the Jefferson Lab polarized ${ }^{3} \mathrm{He}$ collaboration. 


\subsection{Beam characterization}

The Jefferson Lab accelerator delivers up to $100 \mu \mathrm{A}$ of beam polarized at about $80 \%$. The beam polarization, known at the $3 \%$ level, is measured by Mott, Møller and Compton polarimeters. The Compton polarimeter provides continuous monitoring. The beam helicity is flipped at 1 or $30 \mathrm{Hertz}$ and a half wave plate can be inserted in the source's laser in order to invert the beam helicity as a systematic check. The beam current is measured at the percent level by a set of cavities sandwiching an Unser monitor. Beam energy and position on target are locked with feedback systems. Finally, the beam energy is measured either by an accurate tracking of the e(p,ep) reaction ("eP" measurement) or by the measurements of the bending of the beam and of the magnetic field in the arc leading the beam into Hall A ("arc" measurement). Both methods reach an accuracy of $2 . \times 10^{-4}$.

\subsection{The polarized ${ }^{3} \mathrm{He}$ target}

The Jefferson Lab ${ }^{3} \mathrm{He}$ target is the core of our neutron spin structure program. The target has been successfully used by four experiments (GDH* [2], $G_{m}^{n}[3], A_{1}^{n}$ [4] and $\left.g_{2}^{n}[5]\right)$. It consists of either a 25 or a $40 \mathrm{~cm}$ glass cylinder of gaseous ${ }^{3} \mathrm{He}$ at about 12 atm (in running conditions). The target length and the high density allow us to keep a reasonable counting rate in spite of the gaseous nature of the target: The luminosity achieved is $10^{36} \mathrm{~cm}^{-1} \mathrm{~s}^{-1}$ for $15 \mu \mathrm{A}$ and a $40 \mathrm{~cm}$ cylinder length. This cylinder is connected to another chamber where the polarization occurs. The target polarization is achieved in three steps:

- Atoms of Rubidium are optically pumped with a set of lasers.

- The $\mathrm{Rb}$ polarization is transmitted to the ${ }^{3} \mathrm{He}$ by spin exchange collisions.

- The polarized ${ }^{3} \mathrm{He}$ enters in the cylinder by diffusion and convection.

The target can be pumped in any in-plane direction by using two sets of Helmholtz coils and two sets of $90 \mathrm{~W}$ lasers. This feature allows efficient gathering of longitudinal and transverse data.

The polarization, ranging from $35 \%$ to $45 \%$ under running conditions, is measured by Nuclear Magnetic Resonance calibrated using two independent techniques: The known proton thermal polarization (water calibration) and the Electron Paramagnetic Resonance. Both calibrations are consistent. The relative uncertainty on the target polarization is better than $4 \%$.

\section{From ${ }^{3} \mathrm{He}$ to neutron}

The ${ }^{3} \mathrm{He}$ being not in a pure $\mathrm{S}$ wave state, the two proton spins contribute to the nucleus spin. This correction can be accounted for by using the effective polarization of the proton and neutron within the ${ }^{3} \mathrm{He}$ nucleus [6]. Further nuclear effects (Fermi motion and binding) are taken into account within a convolution model. This method has proven reliable for the DIS. It is also expected to be a 
reliable approximation in the resonance region but only for integrated quantities such as sum rules [7]. Our experiments fulfill these conditions so the extraction of the neutron information from the ${ }^{3} \mathrm{He}$ data should be under control. However, it would be highly desirable to have a more complete extraction procedure taking into account other nuclear effects arising at lower $Q^{2}$ such as meson exchange currents and final state interactions. To derive such a method is challenging for theorists but would benefit tremendously the research in neutron spin structure.

\section{The Hall $\mathrm{A} \overrightarrow{{ }^{3} \mathrm{He}}$ experiments}

\subsection{The Hall A GDH* experiment}

As mentioned in the introduction, the transition between the quark/gluon and nucleon/meson degrees of freedom of the strong interaction is not well understood. Its study is one of the chief goals of Jefferson Lab. We will see that the extended Gerasimov-Drell-Hearn $\left(\mathrm{GDH}^{*}\right)$ sum rule is an outstanding tool for studying such a transition.

\subsubsection{The GDH sum rule}

The original GDH sum rule [8] stands at the photon point $\left(Q^{2}=0\right)$. It is (for $\frac{1}{2}$ spin targets):

$$
\int_{\nu_{0}}^{\infty} \sigma^{T T} \frac{d \nu}{v}=-\pi \alpha \frac{\kappa^{2}}{M^{2}}
$$

where $\sigma^{T T}$ is the transverse-transverse interference cross section, $\nu_{0}$ is the pion photoproduction threshold, $\nu$ is the photon energy, $\kappa$ is the anomalous moment of the target and $M$ is its mass. In terms of polarized photoproduction cross sections $\sigma^{T T}=\frac{\sigma^{1 / 2}-\sigma^{3 / 2}}{2}$ with $\frac{1}{2}\left(\frac{3}{2}\right)$ meaning that the photon helicity is anti-aligned (aligned) with the nucleon helicity.

This sum rule is based on solid grounds [13] and is now being tested on the proton at Mainz and ELSA. The preliminary results of these experiments [9] as well as phenomenological models [10], [11] seem to show no gross violation of the sum rule. The neutron situation both for the experimental and phenomenological model predictions is less clear due the lack of a neutron target.

\subsubsection{The extended GDH sum rule}

In 1989, Anselmino et al [12] suggested that a generalization of the GDH integral and the study of its behavior with $\mathrm{Q}^{2}$ will help in understanding the transition from perturbative to non-perturbative QCD. The authors also pointed out the connection between the extended GDH integral and the Bjorken sum rule., The generalization consists of the extension of the integral from photoproduction to 
electroproduction. Indeed, there exist several ways of making such an extension, depending on the choice of the virtual photon flux and how the spin structure function $\mathrm{g}_{2}$ is included. Because of the constraints of the GDH and the Bjorken sum rules, all the definitions are equivalent at the photon point and in the Bjorken limit. They differ however in the intermediate domain (for a review, see [10]).

Among the different GDH* extensions, one - from Ji and Osborn [13] - stands out because it generalizes not only the integral side but the full sum rule. Hence it keeps the checking power of a sum rule that was lost with the former definitions. In addition $\mathrm{Ji}$ and Osborn showed that the Bjorken and GDH sum rules are particular cases of their generalized GDH sum rule. It is written:

$$
\int_{\nu_{0}}^{\infty} G_{1(2)} \frac{d \nu}{v}=\overline{S_{1(2)}}
$$

where $G_{1(2)}$ are the spin structure functions. $\overline{S_{1(2)}}$ are the forward Compton amplitudes with the elastic contribution subtracted. They are calculable for any $Q^{2}$ value using chiral perturbation theory at low $Q^{2}$, higher twist expansion at larger $\mathrm{Q}^{2}$ and eventually lattice QCD calculations.

To summarize, the extended GDH sum rule is a quantity rigorously defined and calculable on the full $\mathrm{Q}^{2}$ range, in particular in the transition region, which makes it an unique tool for understanding the transition between the quark/gluon and nucleon/meson degrees of freedom of the strong interaction.

\subsubsection{The Jefferson Lab Hall A experiment.}

Experiment E94-010 [2] ran at the end of 1998 and aimed to measure the $Q^{2}$ evolution of the integral in the resonance (i.e transition) domain. Its $Q^{2}$ coverage ranges from $0.1 \mathrm{GeV}^{2}$ to $1.0 \mathrm{GeV}^{2}$. The integration is performed up to about $W=2$ $\mathrm{GeV}$. In addition to the resonance domain, the quasi-elastic domain was covered (for nuclear studies) as well as the beginning of the DIS domain in order to link to the E143 and HERMES experiments.

We have measured the inclusive cross sections difference $\sigma^{\uparrow}\left(W, \mathrm{Q}^{2}\right)-\sigma^{\uparrow \downarrow}\left(W, \mathrm{Q}^{2}\right)$ and $\sigma^{\rightarrow}\left(W, Q^{2}\right)-\sigma^{\rightarrow \downarrow}\left(W, Q^{2}\right)$ (The first arrow gives the target polarization direction while the second gives the beam helicity direction) at $15.5^{\circ}$ and six beam energies (0.86 GeV, 1.72 GeV, 2.59 GeV, 3.39 GeV, 4.24 GeV and 5.07 GeV). Linear combinations of these differences provide the two spin structure functions $g_{1}$ and $g_{2} . \sigma^{T T}$ is obtained from these two quantities. Interpolations between the data at different beam energies allow us to get $\sigma^{T T}$ at fixed $\mathrm{Q}^{2}$ in order to integrate it. Let us recall that so far, all the quantities are on ${ }^{3} \mathrm{He}$. The preliminary result of the integration of $\sigma^{T T} / \nu$ is shown in Fig. 1 along with the neutron results. The strong $\mathrm{Q}^{2}$ behavior predicted by the phenomenological models is seen. It appears to be mainly due to the $\mathrm{Q}^{2}$ dependence of $\sigma^{T T}$ at the delta resonance. These data are a benchmark measurement for theoretical calculations (chiral perturbation theory, Lattice QCD and Higher Twist Expansion). They also constrain and improve the phenomenological models describing neutron structure. 


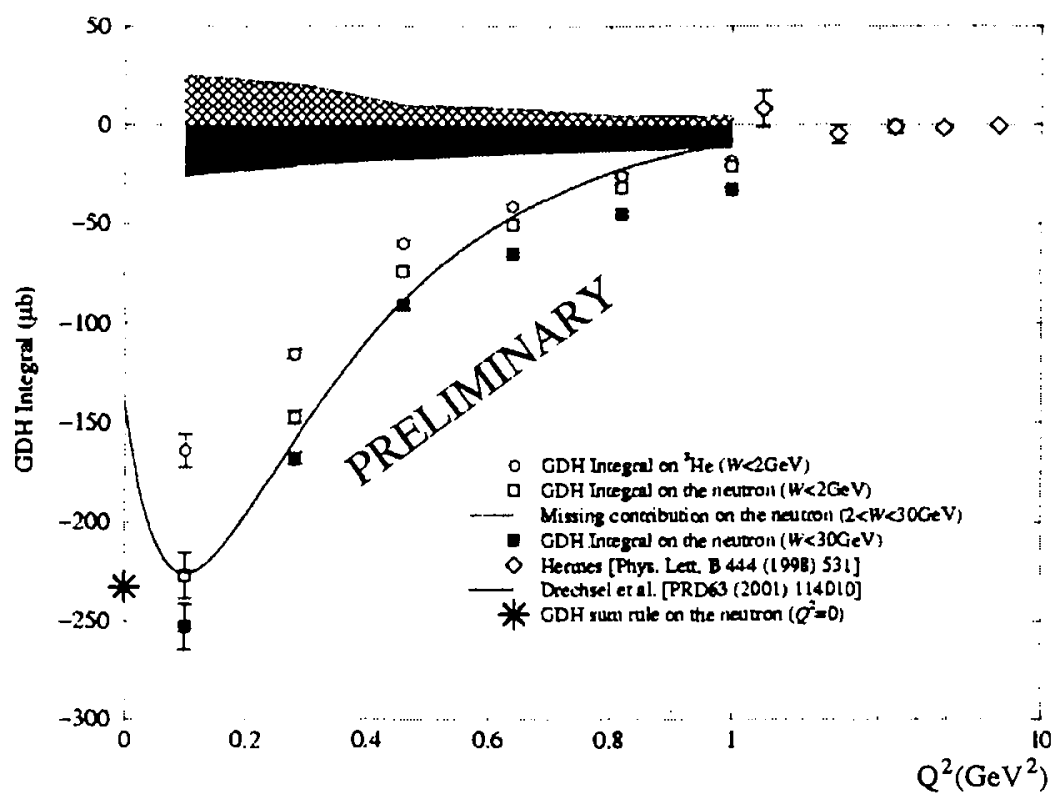

Fig. 1. The GDH* integral on ${ }^{3} \mathrm{He}$ (empty circles) without the quasi-elastic contribution. The neutron (empty squares) is extracted using [7]. The error bars are statistical only. The band below the horizontal axis is an estimate of the contribution of the higher energy reactions, from [11]. Added to our data, we obtain the full squares. The band above the horizontal axis gives the systematic errors. The line is the most recent model describing the GDH $^{*}$ evolution [10] (to be compared with the empty squares). The diamonds are HERMES data [14] (note the log. scale). The star is the original GDH sum rule at the photon point.

\section{2 $\mathrm{GDH}^{*}$ at small $\mathrm{Q}^{2}$}

The previous experiment reached its goal which was to provide accurate data on the $\mathrm{GDH}^{*}$ evolution with $\mathrm{Q}^{2}$ in the intermediate domain. However, these data are inconclusive with regards to the turn around at small $Q^{2}$ predicted by most of the models. This makes any extrapolation of our data to the photon point, in order to check the validity of the original GDH sum rule, hazardous. Hence the necessity arises to complete the previous experiment with a measurement at lower $\mathrm{Q}^{2}$. In addition to a check of the neutron GDH sum rule and chiral perturbation calculations, the measurement of the turn around will provide an additional strong constraint to the phenomenological models.

Experiment E97-110 [15] is scheduled to run in summer 2002. It will take advantage of the new septum magnets of hall $A$ to detect the scattered electrons at angles of $6^{\circ}$ and $9^{\circ}$, reaching an average $Q^{2}$ as low as $0.02 \mathrm{GeV}^{2}$. Its kinematics, compared with the experiment E94-010, are shown in Fig. 2. 


\section{A. Deur}

Kinematic coverage of JLab E94-010 and E97-010

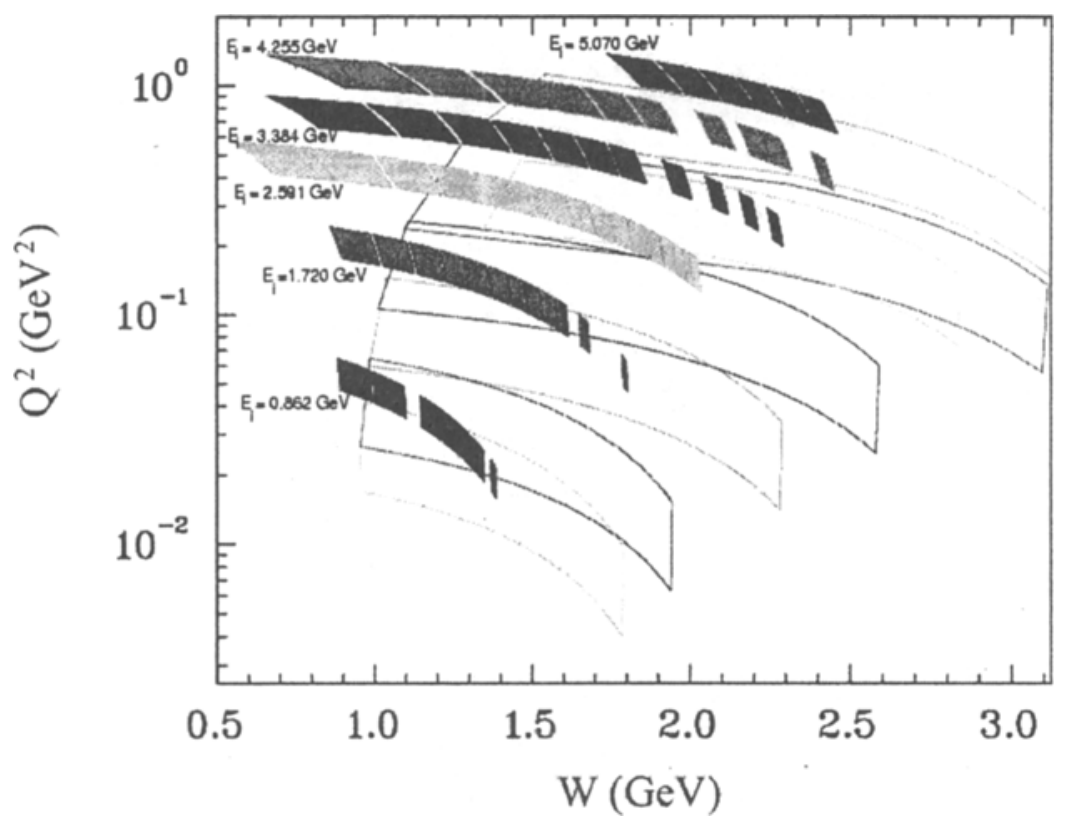

Fig. 2. Kinematics of experiment E94-010 (filled bands) and E97-110 (Empty bands).

\subsection{Higher twist measurements}

Higher twists are quantities beyond the parton model. They are direct effects of the quark-gluon correlations in the nucleon as well as transverse spin and momentum. Results of E155x at SLAC and our E94-010 data show that these quantities are small, even at $\mathrm{Q}^{2}$ lower than $1 \mathrm{GeV}^{2}$. Hence the necessity of a dedicated experiment to measure precisely these quantities. This was the goal of experiment E97-103 [5] which ran in summer 2001. This experiment measured the spin structure function $g_{2}^{n}$ in DIS with unique precision (see Fig. 3). The twist-3 contribution to $g_{2}^{n}$ is extracted using the decomposition:

$$
g_{2}=g_{2}^{w w}+\overline{g_{2}}
$$

where $\overline{g_{2}}$ is purely higher twist (mainly twist 3 ) and $g_{2}^{w w}$ is the leading twist contribution, depending only on $g_{1}$ and given by the Wandzura-Wilczek relation [16]:

$$
g_{2}^{w w}\left(x, \mathrm{Q}^{2}\right)=\int_{x}^{1} g_{1}\left(x^{\prime}, \mathrm{Q}^{2}\right) \frac{d x^{\prime}}{x^{\prime}}-g_{1}\left(x, \mathrm{Q}^{2}\right)
$$

In addition to this experiment, a measurement of the twist 3 matrix element $d_{2}^{n}$ (see for ex. the review [17]) has been proposed [18]. This proposal takes advantage of the unique luminosity of Jefferson Lab in the high $x$, DIS domain. 


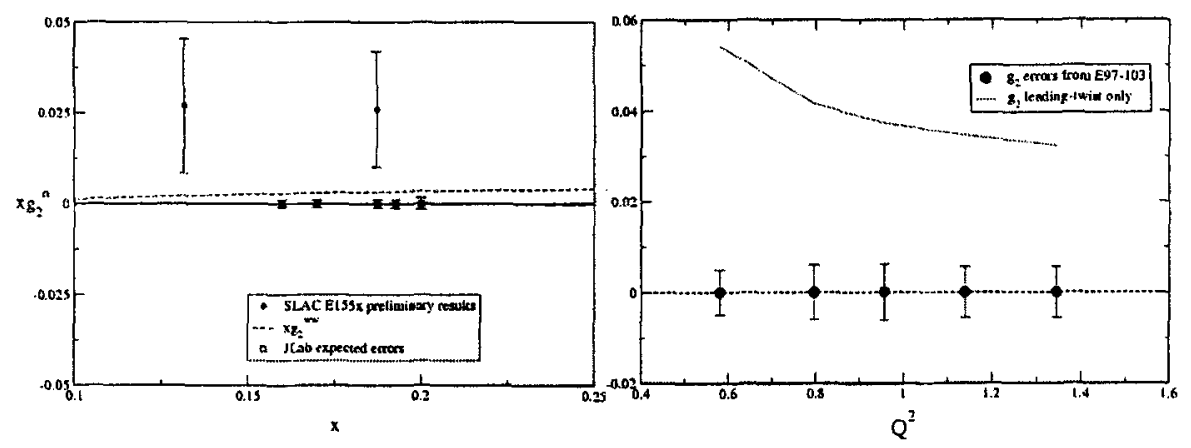

Fig. 3. On the left are shown the expected error bars from experiment E97-103. To be compared with the leading twist contribution to $g_{2}^{n}$ (dashed line) and the E155x preliminary results. Right plot shows the $\mathrm{Q}^{2}$ coverage of E97-103.

\section{4 $A_{1}^{n}(x)$ at large $x$}

As just mentioned, Jefferson Lab is an unique facility to provide reasonable statistics for DIS data at large $x$. The quark constituent model [19], Broken SU(6) symmetry and pQCD calculation all predict that $A_{1}^{n}(x) \rightarrow_{x \rightarrow 1} 1$, although 'with different ways of reaching 1 (see Fig. 4). However, the presently available data at small $x$ are negative and the large $x$ data are inconclusive due to the large size of the error bars. Moreover, naive $\mathrm{SU}(6)$ symmetry and an instanton model [20] predict $A_{1}^{n}(x)$ to be zero or slightly negative at $x=1$. The experiment E99-117 [4] aimed to clarify the situation at large $x(x=0.33,0.52,0.63)$. It ran just before the $g_{2}^{n}$ experiment and the analysis is underway.

\subsection{Bloom-Gilman duality and $A_{1}^{n}(x)$ at larger $x$}

As seen for the $A_{1}^{n}(x)$ experiment, the current $6 \mathrm{GeV}$ limit of Jefferson Lab forbids any measurement above $x=0.6$. While waiting for the $12 \mathrm{GeV}$ upgrade of $\mathrm{JLab}$, another way to access large $x$ is to take data in the resonance region and to use the Bloom-Gilman duality [21] to deduce the behavior of $A_{1}^{n}(x)$ at large $x$. The first goal of experiment E01-012 [22] is to establish the results suggested by the E94-010 experiment: The duality stands for the neutron spin structure functions ${ }^{1}$ ). This will be done by comparing its data with the DIS data of E99-117. Then the measurement will be extended up to $x=0.85$.

Let us note that the previous experiments (apart from $\mathrm{GDH}^{*}$ ) take place in the DIS region where the neutron information extraction is not a problem. In the case of E01-012, the extraction is possible because the result is a sum over the resonances. 


\section{A. Deur}

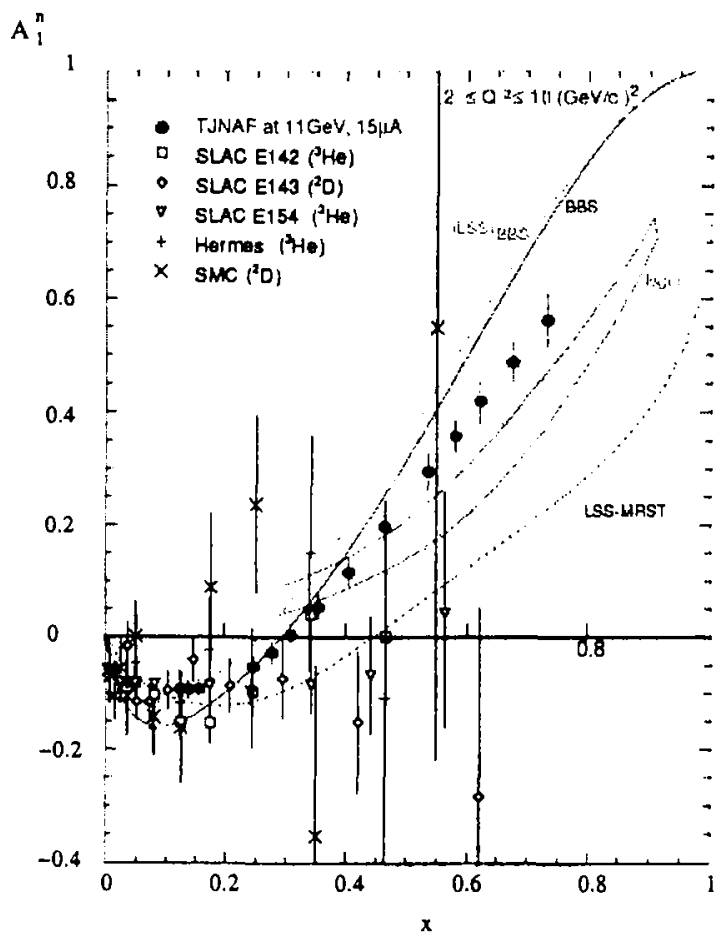

Fig. 4. Projected results for $A_{1}^{n}(x)$ at large x with the $12 \mathrm{GeV}$ upgrade of JLab.

\section{Conclusion and perspectives}

We have seen that, due to the unique luminosity of our experimental set up, the Hall $\mathrm{A}^{3} \overrightarrow{\mathrm{He}}$ program is providing a set of high precision data to study the neutron (and ${ }^{3} \mathrm{He}$ ) structure in a large and complete kinematics domain: Quasi-elastic region, resonance region, DIS domain and DIS domain at large $x$. This information will be critical also for the understanding of the transition between perturbative and non-perturbative QCD. The future upgrade of JLab will provide an extension of this program, particularly the DIS at large $x$ physics. Fig. 4 illustrates the gain provided by such an upgrade. To conclude and set perspectives, it should be noted that $A_{1}^{n}(x)$ and $d_{2}^{n}(x)$ at $12 \mathrm{GeV}$ are leading experiments supporting the JLab upgrade.

\section{References}

[1] B.D. Anderson et al.: Basic Instrumentation for the Hall A at Jefferson Lab. To be published in NIM.

[2] Z. Meziani, G. Cates and J.P. Chen: Jefferson Lab experiment E94-010.

\footnotetext{
1) So far, the duality was checked only on unpolarized structure functions.
} 
[3] H. Gao and O. Hansen: Jefferson Lab experiment E95-01.

[4] J.P. Chen, Z. Meziani and P. Souder: Jefferson Lab experiment E99-117.

[5] T. Averett and W. Korsch: Jefferson Lab experiment E97-103.

[6]. C. Ciofi degli Atti et al.: Phys. Rev. C 48 (1993) R968.

[7] C. Ciofi degli Atti and S. Scopetta: Phys. Let. B 404 (1997) 223-229.

[8] S. Gerasimov: Yad. Fiz. 2 (1965) 598; S.D. Drell and A.C. Hearn: Phys. Rev. Lett. 16 (1966) 908.

[9] GDH2000 proceedings. D. Drechsel and L. Tiator, editors. World Scientific (2001).

[10] D. Drechsel et al.: Phys. rev. D63 114010 (2001).

[11] N. Bianchi and E. Thomas: Phys. Lett. B 450 (1999) 439.

[12] M. Anselmino, B.L. Ioffe and E. Leader: Sov. J. Nucl. Phys. 49 (1989) 136.

[13] X. Ji and J. Osborn: J. Phys G27 (2001) 127.

[14] The HERMES collaboration. Phys. Lett. B444 531 (1998).

[15] J.P Chen, A. Deur and F. Garibaldi: Experiment E97-110.

[16] S. Wandzura and F. Wilczek: Phys. Lett. B72 (1983) 195.

[17] B. Filippone and X. Ji: hep-ph/0101224

[18] X. Jiang and Z. Meziani: Jefferson Lab proposal P01111.

[19] N. Isgur: Phys. Rev. Letter D59 (1999) 034013.

[20] N. Kochelev: hep-ph/9710540.

[21] E.D. Bloom and J.F. Gilman: Phys. Rev. Lett 25 (1970) 1140; Phys. Rev. D4, (1971) 2901.

[22] N. Liyanage, J.P. Chen, S. Choi: Jefferson Lab experiment E01012. 

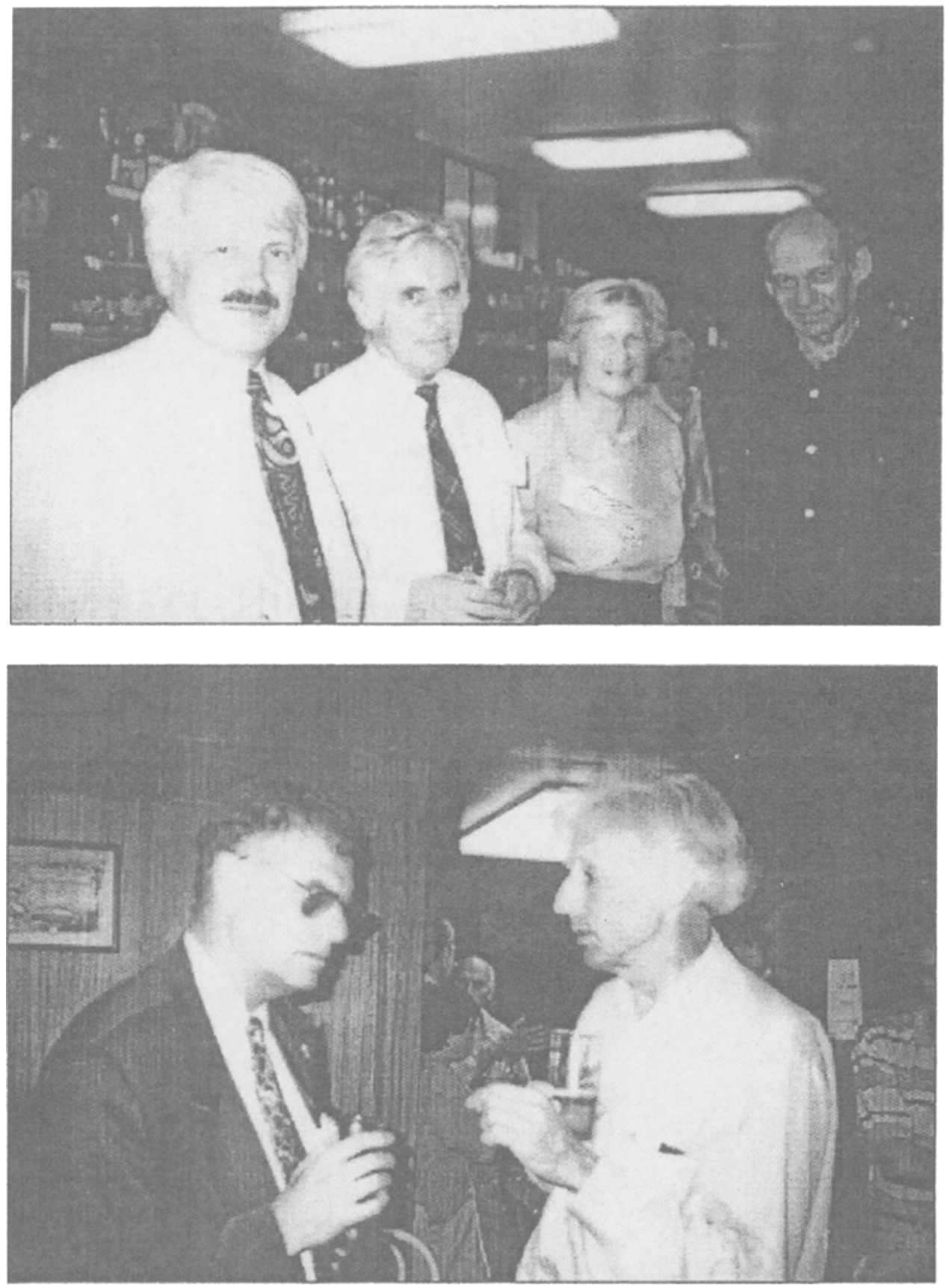